\title{
Tacrolimus- and sirolimus-induced human $\beta$ cell dysfunction is reversible and preventable
}

\author{
Chunhua Dai, ${ }^{1}$ John T. Walker, ${ }^{2}$ Alena Shostak, ${ }^{1}$ Ana Padgett, ${ }^{1}$ Erick Spears, ${ }^{1}$ Scott Wisniewski, ${ }^{1}$ \\ Greg Poffenberger, ${ }^{1}$ Radhika Aramandla, ${ }^{1}$ E. Danielle Dean, ${ }^{1}$ Nripesh Prasad, ${ }^{3}$ Shawn E. Levy, ${ }^{3}$ \\ Dale L. Greiner, ${ }^{4}$ Leonard D. Shultz, ${ }^{5}$ Rita Bottino, ${ }^{6}$ and Alvin C. Powers ${ }^{1,2,7}$ \\ 'Division of Diabetes, Endocrinology and Metabolism, Department of Medicine, and 'Department of Molecular Physiology \\ and Biophysics, Vanderbilt University School of Medicine, Nashville, Tennessee, USA. ${ }^{3} \mathrm{HudsonAlpha} \mathrm{Institute} \mathrm{for}$ \\ Biotechnology, Huntsville, Alabama, USA. ${ }^{4}$ Department of Molecular Medicine, Diabetes Center of Excellence, University \\ of Massachusetts Medical School, Worcester, Massachusetts, USA. ${ }^{5}$ The Jackson Laboratory, Bar Harbor, Maine, \\ USA. ${ }^{6}$ Institute of Cellular Therapeutics, Allegheny-Singer Research Institute, Allegheny Health Network, Pittsburgh, \\ Pennsylvania, USA. ${ }^{7}$ VA Tennessee Valley Healthcare System, Nashville, Tennessee, USA.
}

Posttransplantation diabetes mellitus (PTDM) is a common and significant complication related to immunosuppressive agents required to prevent organ or cell transplant rejection. To elucidate the effects of 2 commonly used agents, the calcineurin inhibitor tacrolimus (TAC) and the mTOR inhibitor sirolimus (SIR), on islet function and test whether these effects could be reversed or prevented, we investigated human islets transplanted into immunodeficient mice treated with TAC or SIR at clinically relevant levels. Both TAC and SIR impaired insulin secretion in fasted and/or stimulated conditions. Treatment with TAC or SIR increased amyloid deposition and islet macrophages, disrupted insulin granule formation, and induced broad transcriptional dysregulation related to peptide processing, ion/calcium flux, and the extracellular matrix; however, it did not affect regulation of $\beta$ cell mass. Interestingly, these $\beta$ cell abnormalities reversed after withdrawal of drug treatment. Furthermore, cotreatment with a GLP-1 receptor agonist completely prevented TACinduced $\beta$ cell dysfunction and partially prevented SIR-induced $\beta$ cell dysfunction. These results highlight the importance of both calcineurin and mTOR signaling in normal human $\beta$ cell function in vivo and suggest that modulation of these pathways may prevent or ameliorate PTDM.

Authorship note: CD and JTW contributed equally to this work.

Conflict of interest: DLG is a consultant and receives research support from The Jackson Laboratory.

Copyright: () 2020, American Society for Clinical Investigation.

Submitted: June 4, 2019 Accepted: November 20, 2019 Published: January 16, 2020.

Reference information: /CI Insight. 2020;5(1):e130770.

https://doi.org/10.1172/jici. insight.130770.

\section{Introduction}

The development of effective immunosuppressive agents has enabled organ and cell transplantation to become a life-saving medical achievement. However, a number of potential adverse events following transplantation threaten the health of both the patient and the graft, including posttransplantation diabetes mellitus (PTDM) (1). PTDM affects between $7 \%$ and $74 \%$ of transplantation patients (with the variance typically being attributed to center-specific differences in pretransplant screenings and diagnostic tests) (2). Furthermore, the risk for PTDM progressively increases in the posttransplantation period, with PTDM incidence increasing linearly with time (3). PTDM is associated with reduced graft function, increased graft loss, and increased patient mortality, making it both a common and significant complication (4). In PTDM, both reduced insulin secretion and impaired insulin action through the development or worsening of insulin resistance have been proposed as mechanisms (2). The pathogenesis of PTDM is likely multifactorial and shares type 2 diabetes genetic risk factors $(2,5)$. Since treatment with immunosuppressive agents tacrolimus (TAC) and sirolimus (SIR) is associated with PTDM (6-9), elucidating the effect of these immunosuppressive agents on human $\beta$ cells may aid the understanding of the pathophysiology and progression of PTDM.

TAC inhibits calcineurin, a calcium/calmodulin-dependent phosphatase that controls activation of the nuclear factor of activated T cells (NFAT) transcription factors. Calcineurin inhibition leads to immunosuppression by preventing $\mathrm{T}$ cell activation and cytokine production (10). The calcineurin/NFAT pathway is also active in other cell types, and inhibition in these cells is thought to mediate the various toxicities of TAC (10). In pancreatic $\beta$ cells, calcineurin/NFAT signaling is thought to positively regulate insulin 
secretion and, in juvenile human islets, $\beta$ cell proliferation $(11,12)$. Furthermore, TAC treatment has been shown to increase $\beta$ cell apoptosis in cultured human islets $(13,14)$.

In contrast, SIR inhibits mTOR, a key regulator of cell metabolism, growth, and proliferation (15). Inhibition of mTOR leads to immunosuppression by preventing $\mathrm{T}$ and $\mathrm{B}$ cell expansion (16). Like the calcineurin/NFAT pathway, mTOR signaling is crucial in many cell types, and its inhibition has adverse effects, including disruption of glucose homeostasis. In insulin-sensitive tissues, SIR treatment disrupts insulin signal transduction, causing insulin resistance $(2,17)$. In mouse islets and human islets in vitro, SIR treatment reduces insulin secretion and decreases $\beta$ cell survival and proliferation $(18,19)$.

While both TAC and SIR appear to have effects on $\beta$ cells, existing studies have largely been performed in vitro or in murine models and have used a wide range of drug doses and treatment schedules that may not be clinically relevant. Importantly, mouse islets show a number of key differences to human islets, including in their stress response, proliferation rates, and both basal and stimulated insulin secretion (2022). In addition, studies of human islets in vitro have been limited to a few days, not mimicking the clinical situation in humans. Thus, findings from prior studies are difficult to translate to patients with PTDM. We therefore sought to clarify the $\beta$ cell effects of TAC or SIR treatment using human islets in an in vivo transplant model that mimics the clinical setting and drug exposure. Here, we show that TAC or SIR treatment at clinically relevant levels leads to $\beta$ cell dysfunction related to multiple islet effects. We also demonstrate that TAC- or SIR-induced $\beta$ cell effects are reversible and that they can, at least partially, be prevented with concurrent glucagon-like peptide-1 receptor (GLP-1R) agonist treatment.

\section{Results}

TAC and SIR impair insulin secretion from human $\beta$ cells at clinically relevant levels in vivo. To investigate immunosuppressive drug exposure in a system that would mimic the human islet response in vivo, we used a human islet transplant model in the immunosuppressed NOD.Cg-Prkdc scid $I l 2 r g^{t m l W j i} / \mathrm{Sz}$ (NSG) mouse, which has deficiencies in both innate and adaptive immunity (23). We first examined the pharmacokinetics of TAC and SIR in the NSG mouse and defined dosing regimens that achieved therapeutic drug levels (TAC, 5-20 ng/mL; SIR, 5-20 $\mathrm{ng} / \mathrm{mL}$; refs. 24, 25). Surprisingly, the half-life of TAC in the NSG mouse is much shorter than in humans (2 hours versus 12 hours); thus, we utilized $0.25 \mathrm{mg} / \mathrm{kg}$ /day delivered by an implanted osmotic pump to achieve clinically relevant levels (Figure 1A and Supplemental Figure 1A; supplemental material available online with this article; https://doi.org/10.1172/jci.insight.130770DS1). For SIR treatment, we found that drug clearance varied drastically between mouse strains, highlighting the importance of robustly characterizing dosing regimens in each model when studying these drugs in mice. Ultimately, $0.2 \mathrm{mg} / \mathrm{kg}$ of SIR delivered via i.p. injection every 72 hours achieved clinically relevant concentrations in the NSG mouse (Figure 1B and Supplemental Figure 1B). These doses are much lower than used in prior studies, which did not report levels of these drugs; this raises questions about the clinical relevance and translational potential of prior findings.

Two weeks following engraftment of human islets, mice began treatment with TAC, SIR, or saline for 4 weeks (Figure 1C). Human islet preparations were analyzed for viability, purity, and function by perifusion analysis; only islets that passed stringent quality control were used for subsequent studies (Supplemental Table 1) (26). We verified targeting of the mTOR pathway by showing that SIR treatment drastically reduced ribosomal protein S6 phosphorylation at 2 critical motifs, Ser235/236 and Ser240/244 in human grafts (Supplemental Figure 2, A-H). Interestingly, TAC also decreased S6 phosphorylation.

Both TAC-treated and SIR-treated mice showed impaired glucose handling by glucose tolerance testing (GTT), with SIR treatment showing greater impairment, likely reflecting SIR-induced insulin resistance (17) (aggregate, Figure 1, D and E; individual donors, Supplemental Figure 3). Mice treated with TAC had no change in fasting blood glucose, while mice treated with SIR showed a slightly higher fasting blood glucose (Figure 1F). As changes in blood glucose likely reflect impact on endogenous mouse organ systems and human islet grafts, we evaluated function of the grafts by measuring serum insulin levels using a human-specific insulin assay and normalizing these to the glucose level of the mouse. In fasted mice, SIR treatment did not affect human insulin or the insulin/glucose ratio, while TAC treatment decreased human insulin and the insulin/glucose ratio (Figure 1, G and H; individual donors, Supplemental Figure 4).

After glucose-arginine stimulation, human islets in both TAC and SIR treatment groups secreted less insulin compared with saline-treated animals (Figure 1, I-K; individual donors, Supplemental Figure 4). SIR-treated mice had both higher blood glucose and human insulin than TAC-treated mice. To assess for direct effects on islets, we cultured human islets in vitro with clinically relevant doses of TAC or SIR (20 
A
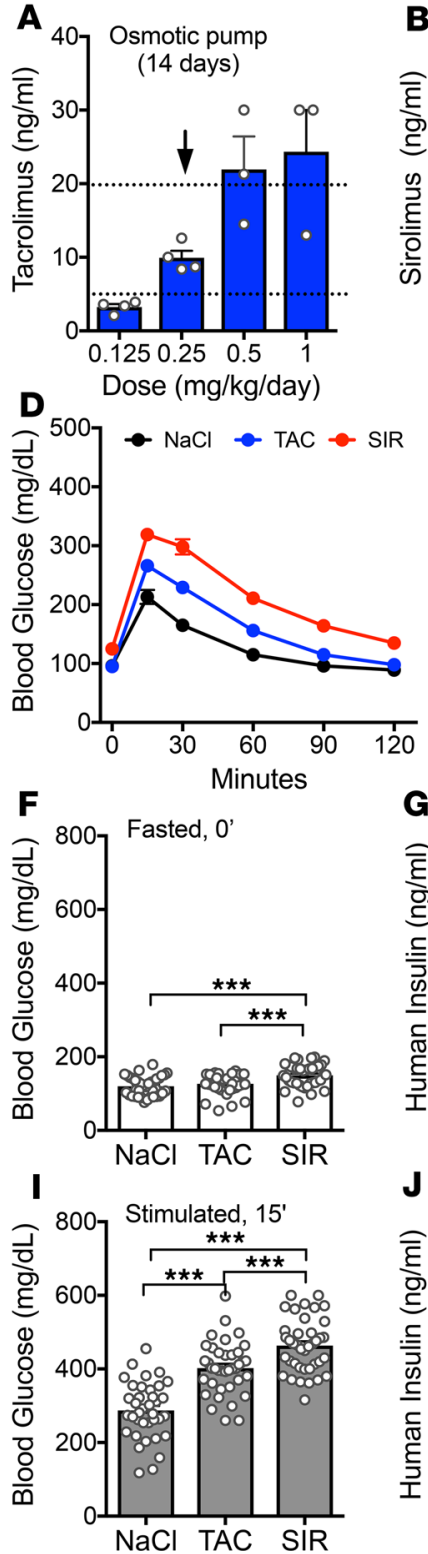

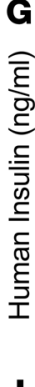

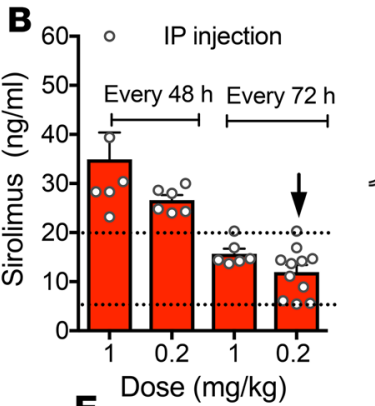

C

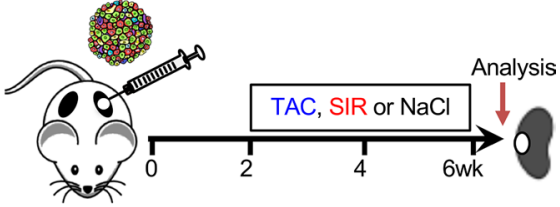

E
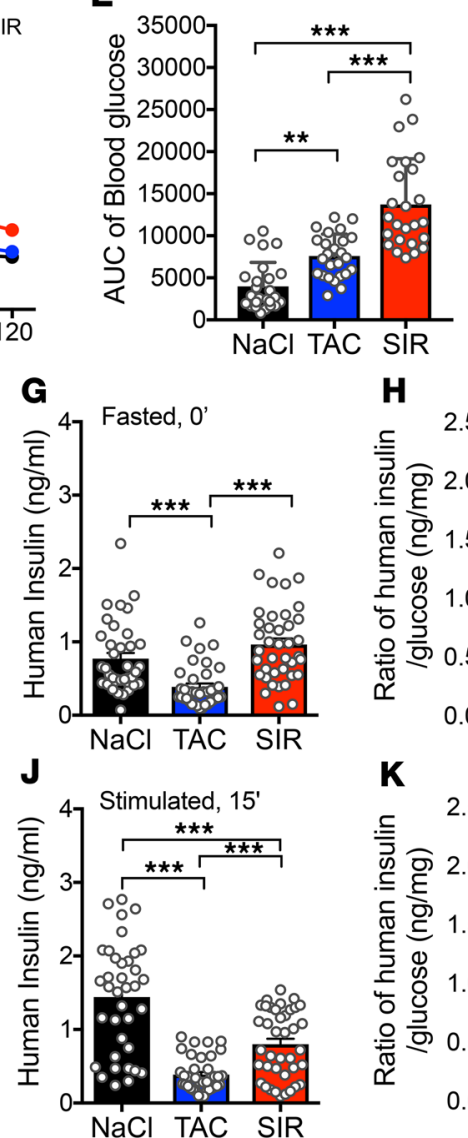

\section{H}

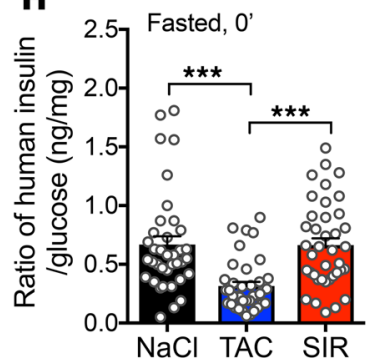

$\mathbf{K}$

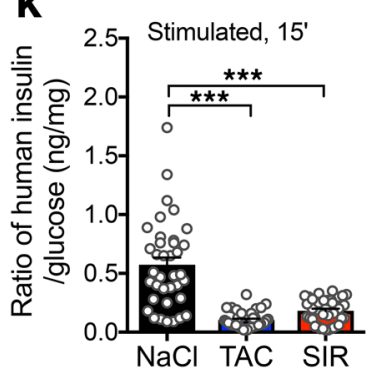

Figure 1. TAC and SIR treatment impairs $\beta$ cell function of human islets in vivo. ( $A$ and $B$ ) TAC (A) and SIR (B) trough levels in NSC mice after 14 days of treatment with various doses and interval times. Dotted lines indicate clinical therapeutic dose ranges, and black arrows indicate doses chosen for this study. (C) Schematic of in vivo experimental design. ( $\mathbf{D}$ and $\mathbf{E}$ ) Glucose tolerance test (GTT) in mice after 4-week treatment (D) and AUC calculations (E; $n=25$ samples/ treatment from 5 donors, donors 3-7; individual donor data shown in Supplemental Figure 3). (F-K) Fasted (6 hours) and 15 minutes after glucose and arginine stimulation blood glucose ( $\mathbf{F}$ and $\mathbf{I}$ ), human insulin levels (G and J), and human insulin/blood glucose ratio ( $H$ and K) ( $n=37-39$ samples/treatment from 7 donors, donors 1-7; individual values and donor data shown in Supplemental Figure 4). ${ }^{*} P<0.05$, ${ }^{* *} P<0.01 ;{ }^{* *} P<0.001$. Error bars indicate \pm SEM. One-way ANOVA followed by Tukey multiple comparisons test was used for analysis of statistical significance.

$\mathrm{ng} / \mathrm{mL}$ ) for 1, 24, and 48 hours and found that, after 48 hours of exposure, insulin secretion was inhibited at both basal $(5.6 \mathrm{mM})$ and high $(16.7 \mathrm{mM})$ glucose (Supplemental Figure 5). These results indicate that, at clinically relevant levels, both SIR and TAC directly affect human $\beta$ cells in vivo by impairing insulin secretion in the stimulated and/or fasted state and that this could be a significant contributor to PTDM.

$T A C$ and SIR treatment do not affect $\beta$ cell proliferation or apoptosis in vivo. To investigate mechanisms underlying the human $\beta$ cell dysfunction induced by TAC or SIR treatment, we harvested islet grafts for analysis. As both the calcineurin and mTOR pathway are involved in the regulation of $\beta$ cell mass $(11-14,19)$, we analyzed $\beta$ cell proliferation by $\mathrm{Ki} 67$ but found low proliferation rates $(<0.5 \%)$ with no differences between treatment groups (Supplemental Figure 6, A and C). Likewise, we assessed for apoptosis by the TUNEL assay; however, apoptotic $\beta$ cells in the grafts were extremely rare, with no difference between treatment groups (Supplemental Figure 6, B and D), indicating that neither TAC nor SIR treatment for 4 weeks at clinically relevant doses affect $\beta$ cell proliferation or apoptosis in human islets in vivo.

TAC and SIR treatment increase amyloid formation and macrophages in human islet grafts. Amyloid deposition in islets, an important pathologic feature in type 2 diabetes, may be a cause or a hallmark of dysfunctional islets (27). Because transplanted human islets form amyloid deposits and this is exacerbated by stressors such as insulin resistance (21), we assessed the grafts for amyloid deposition. Interestingly, we found that 
individual donors varied greatly in the degree of amyloid deposition seen and, thus, report values normalized to the saline-treated control. We found that TAC or SIR treatment increased amyloid deposition in the transplanted human islets (Figure 2, A and B, normalized; Supplemental Table 2, nonnormalized data points), supporting the concept of TAC- and SIR-induced $\beta$ cell dysfunction. Surprisingly, both SIR and TAC treatment also increased the number of $\mathrm{CD} 45^{+}$cells within the graft area (Supplemental Figure 7, A and $\mathrm{B}$ ); these cells were primarily macrophages (as marked by IBA1) and of human origin (human-specific CD45), indicating that these are likely islet-resident macrophages (Supplemental Figure 8, A and B). Islet macrophages play both supportive and detrimental roles within the islet, depending on the context (28-30). The functional effects of TAC or SIR treatment on islet-resident macrophages have not been studied; however, there is evidence that inhibition of the mTOR or NFAT pathways can activate resident macrophages, as opposed to the well-characterized suppression seen in T cells (31-34). Furthermore, amyloid deposits can stimulate islet macrophages to produce IL-1 $\beta$, which can inhibit $\beta$ cell function $(35,36)$.

TAC and SIR treatment disrupt insulin processing and $\beta$ cell granule formation and lead to broad transcriptional dysregulation. To assess for structural alterations in the human $\beta$ cell, we analyzed the grafts by electron microscopy and found fewer $\beta$ cell insulin granules in TAC- and SIR-treated grafts (Figure 2, C and D), suggesting that TAC- or SIR-induced dysfunction is related to impaired mature insulin granule formation, a critical process in coordinated insulin release.

To examine the transcriptional consequences of TAC or SIR treatment, we assessed by reverse transcription PCR (RT-PCR) the expression of genes encoding key molecules involved in $\beta$ cell metabolism (INS, GCK, $S L C 2 A 1), \beta$ cell-enriched transcription factors (PDX1, MAFA, MAFB, NKX6.1), the NFAT family (NFATC1-4), and mTOR signaling (MTOR, RPTOR, RPS6KB1, RPS6) (Supplemental Figure 9, A-D). There were no changes in any of these transcripts in TAC- or SIR-treated grafts.

To obtain a broad view of the transcriptional landscape, we then performed unbiased RNA sequencing (RNA-seq) of the human islet grafts (Supplemental Figure 9, E and F). Gene set pathway analyses of differential gene expression by DAVID (37) within TAC- or SIR-treated grafts revealed enrichment of multiple gene lists related to 3 broad categories: extracellular matrix, ion/calcium flux, and peptide processing (Figure 2, E-G, and Supplemental Tables 3 and 4). Alterations in the extracellular matrix are consistent with the increased amyloid deposits and immune infiltration and with associated increases in inflammatory-related transcripts across both TAC and SIR treatment groups (Supplemental Figure 7, E and F). Dysregulation of genes related to the handling of calcium and other ion flux suggests impaired signal transduction related to stimulated insulin secretion. Finally, alteration of genes related to key insulin processing events such as cleavage at dibasic sites and the formation of disulfide bonds suggests a defect in mature insulin formation. To test this, we analyzed fasting human proinsulin levels in TAC- and SIR-treated mice (Figure $2 \mathrm{H})$. We found elevated human proinsulin/insulin ratios, a marker of $\beta$ cell stress and an early event in the progression of $\beta$ cell dysfunction (38).

In summary, these data indicate that both TAC and SIR, at clinically relevant doses, do not affect regulation of $\beta$ cell proliferation but have broad effects on human islets, including impaired insulin secretion, impaired insulin processing and $\beta$ cell granule formation, and increased amyloid deposition.

Human $\beta$ cell dysfunction reverses after 4-week withdrawal of TAC or SIR. To determine if TAC- or SIR-induced $\beta$ cell dysfunction is reversible, we treated mice transplanted with human islets ( 2 independent donors) with TAC or SIR for 4 weeks and then withdrew treatment and assessed $\beta$ cell function (Figure $3 \mathrm{~A})$. The ability to withdraw treatment and monitor islet function highlights the value of this model, as this would not be possible in humans treated with these agents following organ or cell transplantation. With 4 weeks of TAC or SIR treatment, we saw impaired human graft function, as before (Figure 3, B-E; Supplemental Figure 10, A-D; and Supplemental Figure 11, B, D-G). However, 4 weeks after withdrawal of treatment, we saw a complete normalization of both glucose handling and human $\beta$ cell function in fasted and stimulated states (Figure 3, F-I; Supplemental Figure 10, E-H; Supplemental Figure 11, C and H-K). This suggests that dysfunction induced by TAC or SIR is not permanent and highlights that human $\beta$ cells can recover normal function if such stressors are removed.

Following withdrawal of TAC or SIR, grafts were harvested and analyzed. Compared with grafts from the same treatment group after 4 weeks of treatment, amyloid deposition was higher in the saline-treated control grafts, was stable in TAC-treated grafts, and was lower in SIR-treated grafts (Figure 3, J and K). Thus, after 4 weeks of withdrawal from treatment, amyloid deposition in grafts was similar between all treatment groups. Furthermore, we found that the proportion of immune cells in TAC- and SIR-treated grafts was similar to saline-treated controls after the withdrawal period (Supplemental Figure 7C). Overall, 

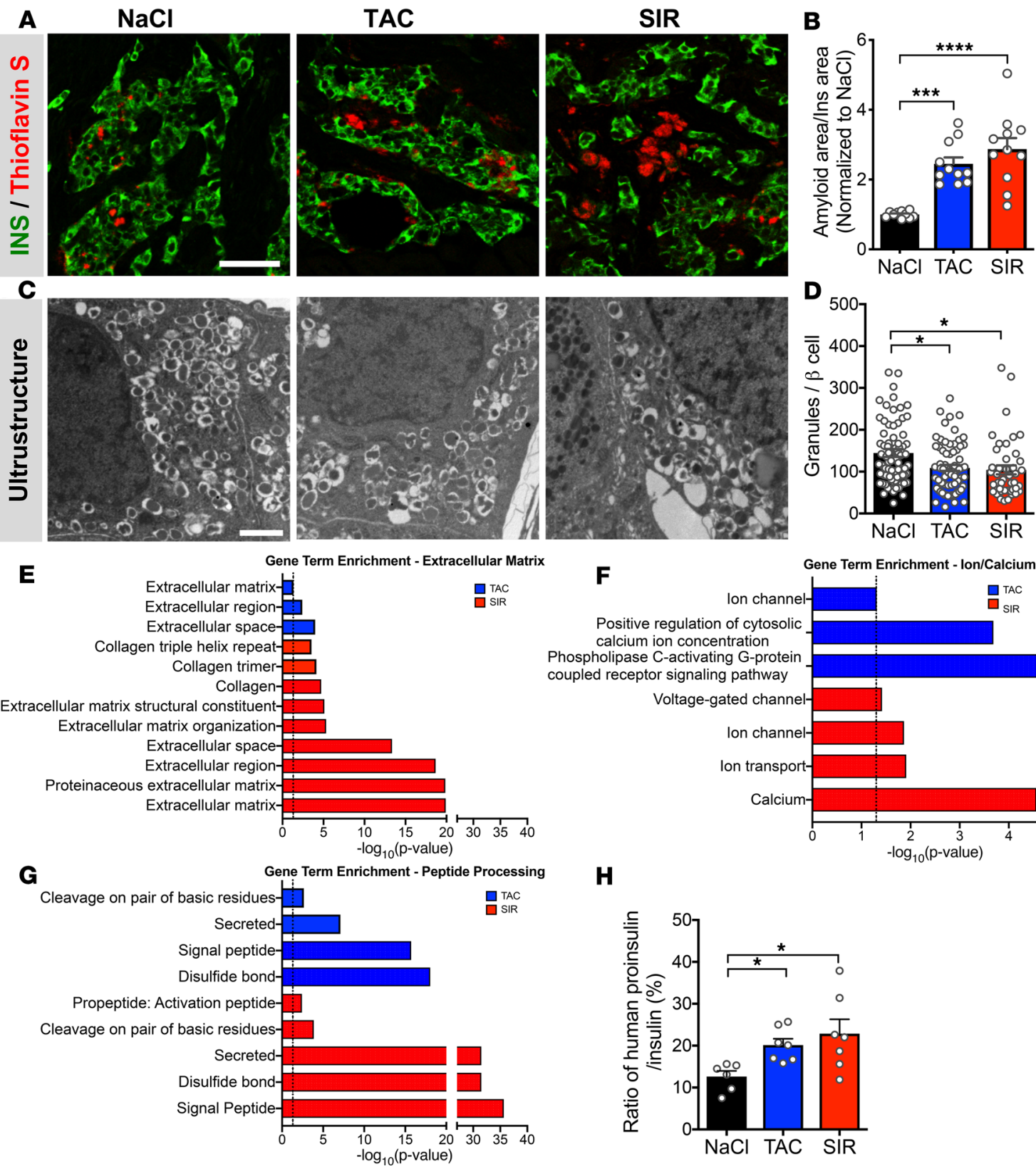

Figure 2. Mechanisms of TAC- and SIR-induced $\boldsymbol{\beta}$ cell dysfunction of human islets in vivo. (A and B) Representative images of amyloid deposits, indicated by thioflavin S (red), in human grafts labeled with insulin (INS, green) (A) and quantification (B) of thioflavin S area/insulin area normalized to the $\mathrm{NaCl}$-treated ratio for that donor to account for donor differences ( $n=10-11$ grafts/treatment from 5 donors; each point represents $1 \mathrm{graft}$, with 5-6 sections analyzed per graft). See Supplemental Table 2 for raw amyloid/insulin area data. Scale bar: $50 \mu \mathrm{m}$ applies to all amyloid images. (C and D) Representative EM images of $\beta$ cells (C) and quantification (D) of granules per $\beta$ cell in human grafts from each drug treatment. Scale bar: $1 \mu \mathrm{m}$ applies to all EM images. (E-G) DAVID gene set enrichment for terms related to extracellular matrix (E), ion/calcium flux (F), peptide processing (G). Note that the $x$-axis scale is different in F. See Supplemental Tables 3 and 4 for full lists from DAVID analysis. $P$ value plotted are the Benjamini Hochberg corrections of 0.05 for FDR, and the dotted line corresponds to $P=0.05$. (H) Ratio of human proinsulin/insulin ( $\mathrm{NaCl}, n=6 ; \mathrm{TAC}, n=7$; and SIR, $n=7$ fasted samples from donor 7). ${ }^{*} P<0.05,{ }^{* *} P<0.001,{ }^{* * *} P<0.0001$. Error bars indicate \pm SEM. One-way ANOVA followed by Tukey multiple comparisons test was used for analysis of statistical significance.

these data indicate that these markers of islet dysfunction can normalize if the treatment is stopped and suggest that amyloid deposits may be cleared if islet insults are removed.

Exendin-4 treatment protects human $\beta$ cells from TAC-induced dysfunction and partially ameliorates SIR-induced dysfunction. While it is helpful to know that the effects of TAC and SIR on human islets can be reversed with withdrawal, these drugs are indispensable in achieving chronic immune suppression in transplant patients. Thus, withdrawal of therapy is not a viable option. To assess ways to protect human islets from 

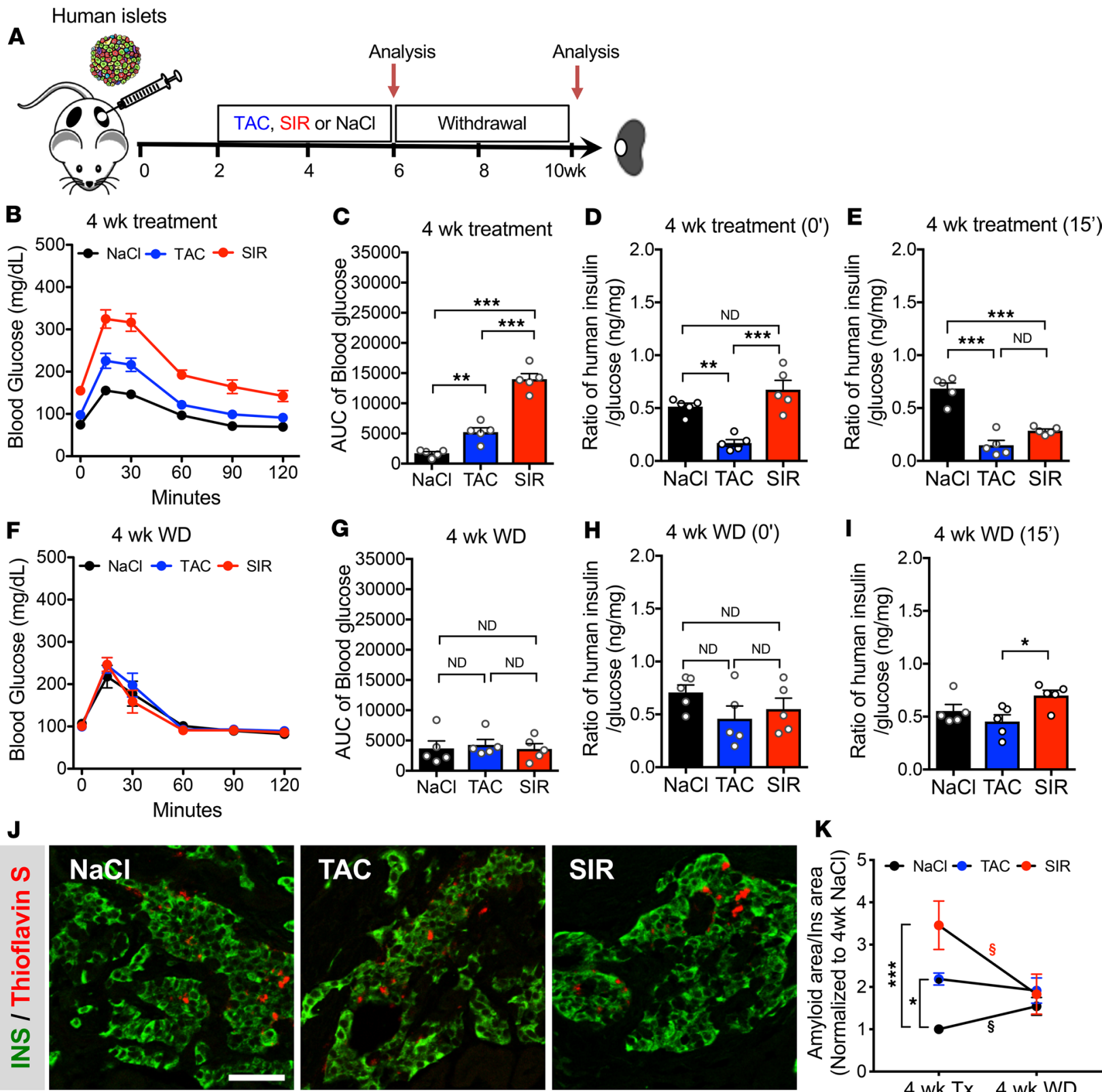

$\mathbf{K}$

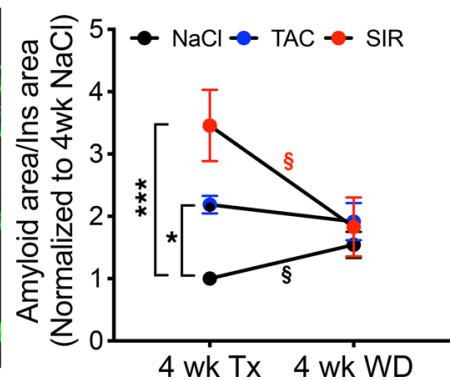

Figure 3. Impaired $\boldsymbol{\beta}$ cell function by either TAC or SIR normalizes after $\mathbf{4}$ weeks of withdrawal. (A) Schematic of experimental design. Mice were given NaCl, TAC, or SIR for 4 weeks, followed by withdrawal (WD) for 4 weeks. (B, C, F, and C) GTT and AUC after treatment with TAC or SIR for 4 weeks (B and C) or 4 weeks after withdrawal (F and $\mathbf{C})$. (D, E, H, and I) Human insulin/blood glucose ratios at 0 and 15 minutes of glucose-arginine stimulation after 4 weeks of treatment ( $\mathbf{D}$ and $\mathbf{E}$ ) and then 4 weeks after withdrawal ( $\mathbf{H}$ and $\mathbf{I} ; n=5$ samples/treatment; donor 7 ). See Supplemental Figure 10 for individual blood glucose and human insulin measurements. See Supplemental Figure 11 for data from a second donor (donor 6), which is shown separately due to significantly different baseline human insulin. (J) Representative images of amyloid after 4-week drug withdrawal and quantification of amyloid burden after 4 weeks of treatment and after 4 weeks of withdrawal (K; $n=4-5$ grafts/treatment from 2 donors, donors 6 and 7, 2-3 grafts per donor, each donor normalized to 4-week treatment with NaCl see Supplemental Table 2 for raw data). Amyloid data is plotted differently from Figure 2 or 4 to show dynamic nature of this process. Scale bar: $50 \mu \mathrm{m}$ applies to all amyloid images. ${ }^{*} P<0.05$, ${ }^{* *} P<0.01,{ }^{* * *} P<0.001$. Error bars indicate $\pm \mathrm{SEM}$. One-way ANOVA followed by Tukey multiple comparisons test was used for analysis of statistical significance. Unpaired $t$ test was used to compare 4-week treatment with 4-week withdrawal within a group: ${ }^{\$} P<0.05$.

drug-induced dysfunction, we cotreated mice bearing human islets from 2 independent donors with either TAC or SIR and Exendin-4 (Ex-4), a GLP-1R agonist (Figure 4A). GLP-1, an incretin hormone released by the $L$ cells of the intestine, acts through $\mathrm{G}_{\mathrm{s}}$-coupled GLP-1 receptors on $\beta$ cells to activate multiple signaling pathways, including the calcineurin/NFAT pathway (39). This signaling acts to potentiate stimulated insulin secretion and also promote young human $\beta$ cell proliferation (11). GLP-1R agonists are used clinically in individuals with type 2 diabetes and have an excellent safety profile. 
The addition of Ex-4 improved glucose handling in both TAC- and SIR-treated groups (Figure 4, B and C). Since GLP-1 signaling is primarily active in $\beta$ cells in the setting of high glucose, we focused on stimulated graft function. We found that TAC + Ex-4 treatment improved stimulated human insulin and the insulin/glucose ratio compared with TAC treatment alone and that these reached levels comparable with islet-graft function in mice never receiving TAC (Figure 4, D-F). In comparison with SIR treatment alone, SIR + Ex-4 treatment led to a slightly improved insulin/glucose ratio (Figure 4, D-F). Taken together, this indicates that Ex-4 is able to completely rescue TAC-induced $\beta$ cell dysfunction and partially rescue SIR-induced $\beta$ cell dysfunction.

Analysis of amyloid deposition within the grafts revealed that TAC + Ex-4-treated grafts had significantly less amyloid than TAC alone and was similar to that of control grafts (Figure 4, G and H). SIR + Ex-4 cotreatment showed a partial reduction in amyloid deposition compared with SIR treatment alone (Figure 4, G and H). Interestingly, Ex-4 cotreatment with TAC or SIR showed only mild reductions in numbers of $\mathrm{CD} 45^{+}$cells within the grafts (Supplemental Figure 7D). Overall, these data are consistent with the functional improvements and demonstrate that TAC- and SIR-induced effects on human islets and amyloid formation can, at least partially, be prevented.

\section{Discussion}

Understanding the effect of immunosuppressive agents on human islets would help elucidate the pathogenesis of PTDM. To study this, we transplanted human islets into immunodeficient mice and treated the mice with clinically relevant levels of 2 commonly used immunosuppressive agents, TAC and SIR. Using this model, we identified several major molecular alterations related to this dysfunction, including reduced $\beta$ cell granules, elevated proinsulin, and increased islet amyloid deposition, which have not been previously reported for $\beta$ cell dysfunction related to immunosuppressive agents. Furthermore, gene pathway enrichment analysis of RNA-seq data revealed that TAC- or SIR-induced broad dysregulation of peptide processing, ion and calcium flux, and extracellular matrix maintenance. Together, these data provide mechanistic insight into TAC- and SIR-induced dysfunction as it relates to PTDM.

With this model, we also demonstrated that TAC- and SIR-induced dysfunction can be reversed with withdrawal of the drug and can be prevented with cotreatment with the GLP-1 agonist Ex-4; these are important findings with clinical implications. Ex-4 and TAC, at least in part, target similar pathways, with TAC inhibiting calcineurin, while GLP-1R signaling in the $\beta$ cell has been shown to lead to calcineurin activation (11). Our data suggest that $\beta$ cell activation of calcineurin through the GLP-1R can overcome inhibition by TAC and, thus, prevent the insulin secretory deficit, though we cannot rule out contribution from additional pathways. Ex-4 and SIR target different pathways, and we noted a partial improvement in human $\beta$ cell health and function. This suggests that targeted mTOR activation in human $\beta$ cells may be an effective approach of protecting human $\beta$ cells from SIR-induced dysfunction.

This study differs from prior reports on the impact of TAC or SIR on islets $(13,14,19)$ in that we studied human islets in vivo and used dosing regimens that achieved clinically relevant levels, thus avoiding toxicity-related effects. In this setting, TAC and SIR affect human $\beta$ cell function without altering $\beta$ cell proliferation or death. This adds to a growing body of literature highlighting differences in how human islets respond in vivo versus in vitro, including in their ability to proliferate, differentiate, and respond to metabolic stressors such as hyperglycemia or dyslipidemia $(21,40-44)$. Thus, these results highlight that in vitro may not always reflect in vivo mechanisms and emphasize the value of studying human islets in a transplantation model.

Our model has a number of advantages, including allowing for studies that would not be possible in humans, while still being able to assess human $\beta$ cells in a complex, in vivo environment. This approach also allows for a comprehensive assessment that takes into account both direct actions of TAC or SIR on the grafts, as well as indirect actions such as SIR-induced insulin resistance. While our model allowed for longer treatment periods than in vitro studies, transplant patients are typically on life-long immunosuppression, and some develop PTDM years after transplant. It is unclear if TAC or SIR exposure over this length of time would have additional effects than what we observed and whether this would be reversible. Another caveat to our approach is that the transplantation procedure may add stress to the islets that would not be present in the pancreas in situ during the development of PTDM. To minimize this, we allowed the islets to engraft for 2 weeks before starting drug treatment, giving the islets time to recover. Furthermore, while the immunosuppressed mouse is the best model to study human islets in vivo, TAC and SIR may have additional indirect effects on human islets by impacting the systemic immune system that are not reflected in our model. Finally, even though we performed all experiments with multiple independent donors, it is 
A

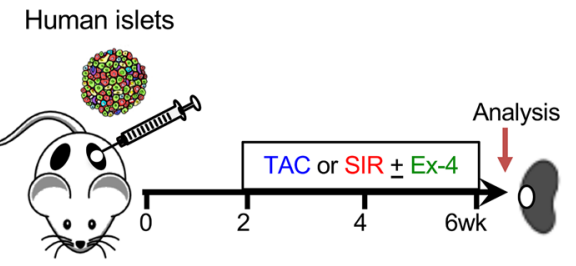

D

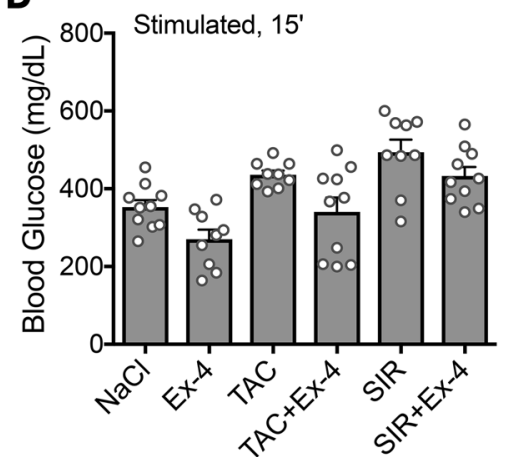

B

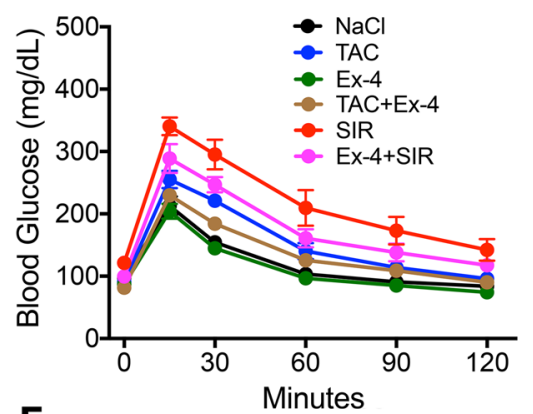

$\mathbf{E}$

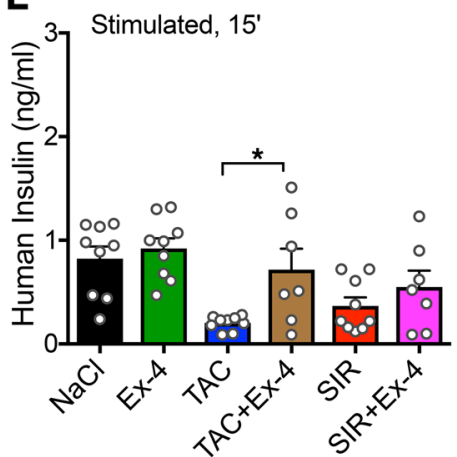

\section{C}

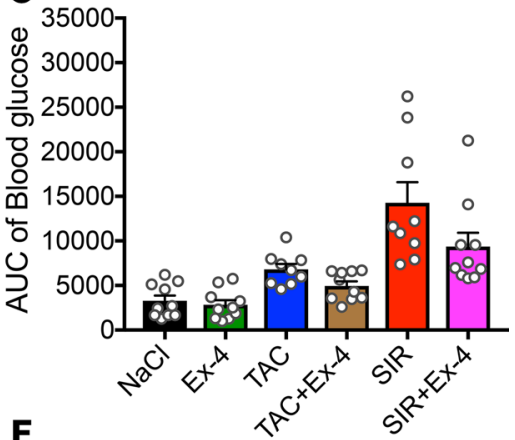

$\mathbf{F}$

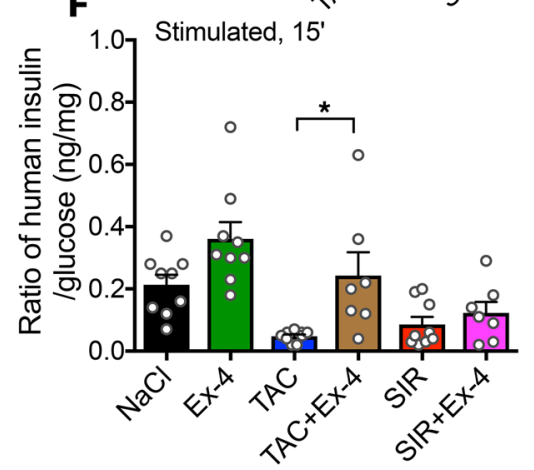

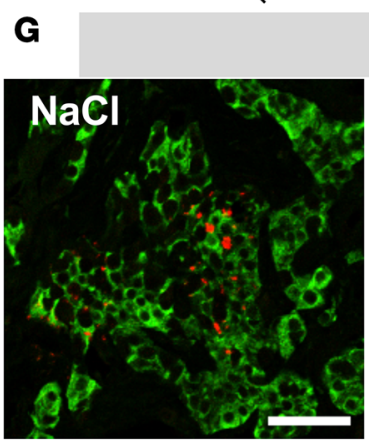

INS / Thioflavin S
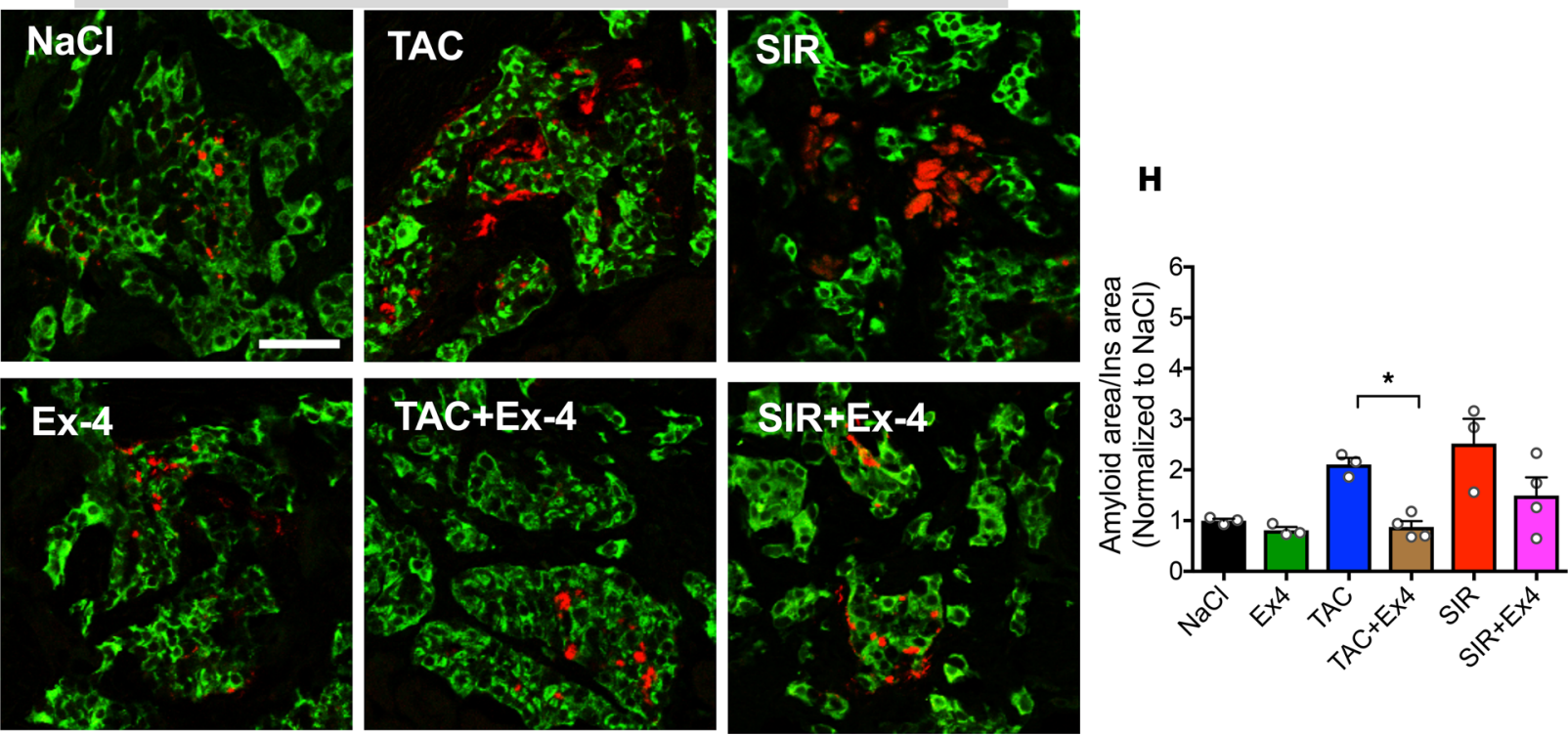

Figure 4. Ex-4 treatment can protect $\beta$ cells from drug-induced dysfunction. (A) Schematic of experimental design with TAC \pm Ex-4 or SIR \pm Ex-4 cotreatment for 4 weeks. (B and C) GTT and AUC treatment with TAC or SIR with or without Ex-4 for 4 weeks. (D-F) Blood glucose, human insulin, and human insulin/blood glucose ratios at 15 minutes after glucose-arginine stimulation ( $n=7-10$ samples/treatment from donors 4 and 5 ). (G and $\mathbf{H}$ ) Representative images (G) of amyloid after 4 weeks of treatment and quantification $(\mathbf{H} ; n=3-4$ grafts/treatment from 2 donors, donors 4 and $5-$ see Supplemental Table 2 for raw data). Scale bar: $50 \mu \mathrm{m}$ applies to all amyloid images. ${ }^{*} P<0.05$. Data represent mean \pm SEM. One-way ANOVA followed by Tukey multiple comparisons test was used for analysis of statistical significance. We highlight only the differences between TAC vs. TAC + Ex-4, or SIR vs. SIR + Ex-4 within panels C-F and $\mathbf{H}$. Full statistical comparisons are shown in Supplemental Table 5.

possible that the genetic and epigenetic variation of human islet donors could play a role in susceptibility to these drugs and PTDM.

PTDM is a major complication after all solid organ and cell transplantation, but clear treatment guidelines for long-term management or prevention are lacking (1). Studies evaluating GLP-1-based therapies in PTDM are limited but indicate that short-term GLP-1 administration can improve insulin secretion (45). 
Furthermore, small retrospective analyses have shown that sitagliptin, a DPP-4 inhibitor that boosts endogenous GLP-1 levels by preventing its breakdown, is safe and efficacious in treating PTDM (2). Our data provide a strong rationale for cotreatment with a GLP-1-based agonist and suggest that clinical trials might test whether GLP-1 agonist treatment may be beneficial in patients treated with TAC or SIR.

In summary, we highlight that TAC- or SIR-induced human islet dysfunction is both reversible with withdrawal of treatment and, at least partially, preventable with cotreatment with a GLP-1R agonist. These studies highlight the importance of both calcineurin/NFAT and mTOR pathways in human $\beta$ cell function and suggest that activation of these pathways in $\beta$ cells may be a useful approach to reduce the incidence of PTDM.

\section{Methods}

Human islets and islet transplantation. Human islets $(n=11$ preparations, Supplemental Table 1$)$ were obtained from Integrated Islet Distribution Network (http://iidp.coh.org/), from Human Pancreas Analysis Program (https://hpap.pmacs.upenn.edu/), or isolated at the Institute of Cellular Therapeutics of the Allegheny Health Network (Pittsburgh, Pennsylvania, USA). Assessment of human islet function was performed by islet perifusion assay on the day of arrival, as previously described $(20,26)$. Male NSG mice (23), age 12-18 weeks, were used for transplantation. Human islets were transplanted under the kidney capsule as previously described $(11,21)$. Each mouse received 1500 IEQ, and islets from a given donor were divided evenly among all 3 treatment groups.

This study used data from the Organ Procurement and Transplantation Network (OPTN). The OPTN data system includes data on all donor, wait-listed candidates, and transplant recipients in the US, submitted by the members of the OPTN. The Health Resources and Services Administration (HRSA), US Department of Health and Human Services, provides oversight to the activities of the OPTN contractor. The data reported here have been supplied by United Network for Organ Sharing (UNOS) as the contractor for the OPTN. The interpretation and reporting of these data are the responsibility of the authors and in no way should be seen as an official policy of or interpretation by the OPTN or the US government.

$T A C, S I R$, and Ex-4 administration. Two weeks after human islet transplantation, TAC $(0.25 \mathrm{mg} / \mathrm{kg} /$ day, NDC 0469-3016-01, Astellas Pharma US Inc.) or saline was delivered by micro-osmotic pumps (Alzet, 1004) implanted in recipient NSG mice. SIR $(0.2 \mathrm{mg} / \mathrm{kg}$, NDC 0008-1030-06, Pfizer) or saline was given by i.p. injection every 72 hours. After 4 weeks of drug treatment, the human grafts were removed for subsequent study (Figure 1C). Saline delivered via osmotic pump versus i.p. injection had no effect on glucose, insulin levels, morphology, or cell composition of grafts, and as such, we combined them into 1 control group for data analysis. Blood concentrations of TAC and SIR were measured by Vanderbilt Diagnostic Laboratories. Ex-4 (24 nmol/kg/day, California Peptide Research Inc.) or 1× PBS was also delivered by micro-osmotic pumps (Alzet, 1004) as previously described (11).

Assessment of glucose tolerance and serum insulin. I.p. GTTs were performed after a 6-hour fast, as previously described (21). For glucose-stimulated insulin secretion (GSIS), the mice were fasted for 6 hours and then injected i.p. with glucose/arginine $(2 \mathrm{~g} / \mathrm{kg})$. Blood samples were obtained before ( 0 minutes $)$ and after (15 minutes) injection. In Ex-4 experiments, the mice were injected with Ex-4 (100 $\mu \mathrm{g} / \mathrm{kg}) 30$ minutes before GSIS. Serum human insulin levels were measured as previously described (21).

IHC of human islet grafts. Frozen human islet graft sections ( $5 \mu \mathrm{m}$ thick) were cut and stained as described $(11,21)$. Primary antibodies used in this project are listed in Supplemental Table 6. Apoptosis was assessed by TUNEL (MilliporeSigma, S7165) following the manufacturer's instructions. To assess amyloid deposits, sections from human grafts were first stained with the insulin antibody and then incubated with $0.5 \%$ concentration Thioflavin S (T-1892, MilliporeSigma) in PBS. Images were acquired with an Olympus BX41 fluorescence microscope or a confocal laser-scanning microscope. Positive Ki67 or pS6 $\beta$ cells and areas of Thioflavin S or CD45 were quantified with MetaMorph software.

Electron microscopy. Ultrastructure of $\beta$ cells was studied by transmission electron microscopy (21). Human islet grafts were removed after perfusion and fixed in $2.5 \%$ gluteraldehyde in $0.1 \mathrm{M}$ cacodylate buffer. Samples were subsequently imaged on the Philips/FEI Tecnai T12 microscope at various magnifications.

RNA isolation, $c D N A$ synthesis, and quantitative PCR. Total RNA was extracted from human islet grafts using an RNAqueous RNA isolation kit (Ambion). RNA quality control and quantity assessment (QC/ QA) was performed using Bioanalyzer instrument in the Vanderbilt Function Genomics Shared Resource (FGSR) core lab. cDNA was synthesized using High Capacity cDNA Reverse Transcription Kit (Applied Biosystems, 4368814) according to the manufacturer's instructions. Quantitative PCR (qPCR) was per- 
formed using TaqMan assays and reagents from Applied Biosystems as described (11, 20, 21). Primers used in this project are listed in Supplemental Table 7. SYPL1, SV2A, and CHDA were used for control genes. Relative changes in mRNA expression were calculated by the comparative $\triangle \mathrm{Ct}$ method using Applied Biosystems Stepone Plus System.

$R N A$-seq library preparation, sequencing, and data analysis. A minimum of 500 nanograms of total RNA (RNA isolation as above) was used for downstream RNA-seq applications. Polyadenylated RNAs were isolated using NEBNext Magnetic Oligo d(T)25 Beads. The NEBNext mRNA Library Prep Reagent Set for Illumina (New England BioLabs Inc.) was then used to prepare individually bar-coded next-generation sequencing expression libraries as per manufacturer's recommended protocol. Library concentration was assessed using the Qubit 2.0 Fluorometer, and the library quality was estimated by utilizing a DNA 1000 Chip on an Agilent 2100 Bioanalyzer. Accurate quantification for sequencing applications was determined using the qPCR-based KAPA Biosystems Library Quantification Kit (Kapa Biosystems Inc.). Paired-end sequencing (100 million, 100-bp, paired-end reads) was performed on an Illumina HiSeq2500 sequencer.

Postprocessing of the sequencing reads from RNA-seq experiments for each sample was performed using HudsonAlpha's in-house RNA-seq data analysis pipeline. Briefly, quality control checks on raw sequence data for each sample were performed using FastQC (Babraham Bioinformatics). Raw reads were mapped to the reference $h g 19$ using TopHat v2.0. The alignment metrics of the mapped reads were estimated using SAMtools. Aligned reads were imported to the commercial data analysis platform Avadis NGS (Strand Scientifics). After quality inspection, the aligned reads were filtered on the basis of read quality metrics; reads with a base quality score of less than 30 , alignment score of less than 95, and mapping quality of less than 40 were removed. Remaining reads were then filtered on the basis of their read statistics; missing mates, translocated, unaligned, and flipped reads were removed. The reads list was then filtered to remove duplicates. Samples were grouped, and transcript abundance was quantified for this final read list using Trimmed Means of M-values (TMM) as the normalization method. Output data utilized for all subsequent comparisons were summarized as normalized expression values generated by Avadis NGS. Differential expression of genes was calculated on the basis of fold changes (using the default cut-off of $\geq 1.5$ or $\leq-1.5$ ) observed in comparisons between defined conditions. DAVID v6.8 analysis was performed on the differentially expressed gene list of $1.5 \times$ fold change (37). This data has been deposited in the GEO repository (GSE140230; www.ncbi.nlm.nih. gov/geo/query/acc.cgi?acc=GSE140230)

Statistics. Statistical significance of differences was determined by 1-way ANOVA followed by Tukey multiple comparisons tests or 1 -tailed, unpaired $t$ test. $P<0.05$ was considered statistically significant. Values represent mean \pm SEM. For RNA-seq analyses, $P$ values of the differentially expressed gene list were estimated by z-test using Benjamini Hochberg corrections of 0.05 for FDR.

Study approval. The Vanderbilt University IRB does not classify deidentified human pancreatic specimens as human subject research. All animal studies were approved by the Vanderbilt IACUC.

\section{Author contributions}

$\mathrm{CD}$ and $\mathrm{ACP}$ designed the experiments. JTW, CD, ES, and ACP wrote the manuscript. CD, AS, AP, SW, GP, JTW, NP, and RB performed experiments or analyzed the data. JTW, CD, EDD, RA, SEL, DLG, LDS, RB, and ACP edited the manuscript. All authors reviewed the final version.

\section{Acknowledgments}

We thank the organ donors and their families for their invaluable donation and the International Institute for Advancement of Medicine (IIAM), Organ Procurement Organizations, and National Disease Research Interchange (NDRI) for their partnership in studies of human pancreatic tissue for research. Human pancreatic islets were provided by the NIDDK-funded Integrated Islet Distribution Program at the City of Hope (NIH grant 2UC4 DK098085). We thank Asma Khaled Aljaberi for her technical assistance. This research was performed using resources and/or funding provided by the NIDDK-supported HIRN (RRID:SCR_014393; https://hirnetwork.org; UC4 DK104211, UC4 DK104218, DK108120, and DK112232); by DK106755, DK72473, DK89572, DK97829, DK94199, T32GM007347, F30DK118830, and DK20593; and by grants from JDRF, The Leona M. and Harry B. Helmsley Charitable Trust, and the Department of Veterans Affairs (BX000666). 
Address correspondence to: Alvin C. Powers, Vanderbilt University Medical Center, 7465 Medical Research Building IV, 2215 Garland Avenue, Nashville, Tennessee 37232-0475, USA. Phone: 615.936.7678; Email: al.powers@vumc.org.

1. Sharif A, et al. Proceedings from an international consensus meeting on posttransplantation diabetes mellitus: recommendations and future directions. Am J Transplant. 2014;14(9):1992-2000.

2. Shivaswamy V, Boerner B, Larsen J. Post-Transplant Diabetes Mellitus: Causes, Treatment, and Impact on Outcomes. Endocr Rev. 2016;37(1):37-61.

3. Cosio FG, Pesavento TE, Osei K, Henry ML, Ferguson RM. Post-transplant diabetes mellitus: increasing incidence in renal allograft recipients transplanted in recent years. Kidney Int. 2001;59(2):732-737.

4. Davidson J, et al. New-onset diabetes after transplantation: 2003 International consensus guidelines. Proceedings of an international expert panel meeting. Barcelona, Spain, 19 February 2003. Transplantation. 2003;75(10 Suppl):SS3-S24.

5. Pham PT, Pham PM, Pham SV, Pham PA, Pham PC. New onset diabetes after transplantation (NODAT): an overview. Diabetes Metab Syndr Obes. 2011;4:175-186.

6. Ekberg H, et al. Reduced exposure to calcineurin inhibitors in renal transplantation. N Engl J Med. 2007;357(25):2562-2575.

7. Vincenti $F$, et al. Results of an international, randomized trial comparing glucose metabolism disorders and outcome with cyclosporine versus tacrolimus. Am J Transplant. 2007;7(6):1506-1514.

8. Johnston O, Rose CL, Webster AC, Gill JS. Sirolimus is associated with new-onset diabetes in kidney transplant recipients. $J$ Am Soc Nephrol. 2008;19(7):1411-1418.

9. Teutonico A, Schena PF, Di Paolo S. Glucose metabolism in renal transplant recipients: effect of calcineurin inhibitor withdrawal and conversion to sirolimus. J Am Soc Nephrol. 2005;16(10):3128-3135.

10. Macian F. NFAT proteins: key regulators of T-cell development and function. Nat Rev Immunol. 2005;5(6):472-484.

11. Dai C, et al. Age-dependent human $\beta$ cell proliferation induced by glucagon-like peptide 1 and calcineurin signaling. J Clin Invest. 2017;127(10):3835-3844.

12. Heit JJ, et al. Calcineurin/NFAT signalling regulates pancreatic beta-cell growth and function. Nature. 2006;443(7109):345-349

13. Soleimanpour SA, et al. Calcineurin signaling regulates human islet \{beta\}-cell survival. J Biol Chem. 2010;285(51):4005040059.

14. Johnson JD, et al. Different effects of FK506, rapamycin, and mycophenolate mofetil on glucose-stimulated insulin release and apoptosis in human islets. Cell Transplant. 2009;18(8):833-845.

15. Li J, Kim SG, Blenis J. Rapamycin: one drug, many effects. Cell Metab. 2014;19(3):373-379.

16. Thomson AW, Turnquist HR, Raimondi G. Immunoregulatory functions of mTOR inhibition. Nat Rev Immunol. 2009;9(5):324-337.

17. Lamming DW, et al. Rapamycin-induced insulin resistance is mediated by mTORC2 loss and uncoupled from longevity. Science. 2012;335(6076):1638-1643.

18. Barlow AD, Nicholson ML, Herbert TP. Evidence for rapamycin toxicity in pancreatic $\beta$-cells and a review of the underlying molecular mechanisms. Diabetes. 2013;62(8):2674-2682.

19. Nir T, Melton DA, Dor Y. Recovery from diabetes in mice by beta cell regeneration. J Clin Invest. 2007;117(9):2553-2561.

20. Dai C, et al. Islet-enriched gene expression and glucose-induced insulin secretion in human and mouse islets. Diabetologia. 2012;55(3):707-718.

21. Dai C, et al. Stress-impaired transcription factor expression and insulin secretion in transplanted human islets. J Clin Invest. 2016;126(5):1857-1870

22. Hart NJ, Powers AC. Use of human islets to understand islet biology and diabetes: progress, challenges and suggestions. Diabetologia. 2019;62(2):212-222.

23. Shultz LD, et al. Human lymphoid and myeloid cell development in NOD/LtSz-scid IL2R gamma null mice engrafted with mobilized human hemopoietic stem cells. J Immunol. 2005;174(10):6477-6489.

24. Andrews LM, et al. Pharmacokinetic considerations related to therapeutic drug monitoring of tacrolimus in kidney transplant patients. Expert Opin Drug Metab Toxicol. 2017;13(12):1225-1236.

25. Mahalati K, Kahan BD. Clinical pharmacokinetics of sirolimus. Clin Pharmacokinet. 2001;40(8):573-585.

26. Kayton NS, et al. Human islet preparations distributed for research exhibit a variety of insulin-secretory profiles. Am J Physiol Endocrinol Metab. 2015;308(7):E592-E602.

27. Westermark P, Andersson A, Westermark GT. Islet amyloid polypeptide, islet amyloid, and diabetes mellitus. Physiol Rev. 2011;91(3):795-826.

28. Aamodt KI, Powers AC. Signals in the pancreatic islet microenvironment influence $\beta$-cell proliferation. Diabetes Obes Metab. 2017;19 Suppl 1:124-136.

29. Eguchi K, Nagai R. Islet inflammation in type 2 diabetes and physiology. J Clin Invest. 2017;127(1):14-23.

30. Ying W, et al. Expansion of Islet-Resident Macrophages Leads to Inflammation Affecting $\beta$ Cell Proliferation and Function in Obesity. Cell Metab. 2019;29(2):457-474.e5.

31. Fric J, Zelante T, Wong AY, Mertes A, Yu HB, Ricciardi-Castagnoli P. NFAT control of innate immunity. Blood. 2012;120(7):1380-1389.

32. Kang YJ, et al. Calcineurin negatively regulates TLR-mediated activation pathways. J Immunol. 2007;179(7):4598-4607.

33. Weichhart T, Hengstschläger M, Linke M. Regulation of innate immune cell function by mTOR. Nat Rev Immunol. 2015;15(10):599-614.

34. Mercalli A, et al. Rapamycin unbalances the polarization of human macrophages to M1. Immunology. 2013;140(2):179-190.

35. Masters SL, et al. Activation of the NLRP3 inflammasome by islet amyloid polypeptide provides a mechanism for enhanced IL-1 $\beta$ in type 2 diabetes. Nat Immunol. 2010;11(10):897-904.

36. Westwell-Roper CY, Ehses JA, Verchere CB. Resident macrophages mediate islet amyloid polypeptide-induced islet IL-1 $\beta$ production and $\beta$-cell dysfunction. Diabetes. 2014;63(5):1698-1711. 
37. Huang da W, Sherman BT, Lempicki RA. Systematic and integrative analysis of large gene lists using DAVID bioinformatics resources. Nat Protoc. 2009;4(1):44-57.

38. Arunagiri A, et al. Proinsulin misfolding is an early event in the progression to type 2 diabetes. Elife. 2019;8:e44532.

39. Drucker DJ. Mechanisms of Action and Therapeutic Application of Glucagon-like Peptide-1. Cell Metab. 2018;27(4):740-756.

40. Wang $\mathrm{P}$, et al. A high-throughput chemical screen reveals that harmine-mediated inhibition of DYRK1A increases human pancreatic beta cell replication. Nat Med. 2015;21(4):383-388.

41. Wang P, et al. Combined Inhibition of DYRK1A, SMAD, and Trithorax Pathways Synergizes to Induce Robust Replication in Adult Human Beta Cells. Cell Metab. 2019;29(3):638-652.e5.

42. Nair GG, et al. Recapitulating endocrine cell clustering in culture promotes maturation of human stem-cell-derived $\beta$ cells. Nat Cell Biol. 2019;21(2):263-274.

43. Pagliuca FW, et al. Generation of functional human pancreatic $\beta$ cells in vitro. Cell. 2014;159(2):428-439.

44. Rezania A, et al. Reversal of diabetes with insulin-producing cells derived in vitro from human pluripotent stem cells. Nat Biotechnol. 2014;32(11):1121-1133.

45. Halden TA, et al. GLP-1 Restores Altered Insulin and Glucagon Secretion in Posttransplantation Diabetes. Diabetes Care. 2016;39(4):617-624 